\title{
Lack of association between thyroid autoantibodies and parity in a population study argues against microchimerism as a trigger of thyroid autoimmunity
}

\author{
Inge Bülow Pedersen ${ }^{1}$, Peter Laurberg ${ }^{1}$, Nils Knudsen ${ }^{2,3}$, Torben Jørgensen ${ }^{3}$, Hans Perrild ${ }^{2}$, Lars Ovesen ${ }^{4}$ and \\ Lone Banke Rasmussen ${ }^{4}$ \\ ${ }^{1}$ Department of Endocrinology and Medicine, Aalborg Hospital, Aarhus University Hospital, DK-9000 Aalborg, Denmark, ${ }^{2}$ Endocrine Unit, Department \\ of Internal Medicine I, Bispebjerg Hospital, Copenhagen, Denmark, ${ }^{3}$ Research Centre for Prevention and Health, Glostrup University Hospital, \\ Copenhagen, Denmark and ${ }^{4}$ Department of Nutrition, Danish Institute for Food and Veterinary Research, Copenhagen, Denmark
}

(Correspondence should be addressed to I Bülow Pedersen; Email: Ibulow@aas.nja.dk)

\begin{abstract}
Background: Thyroid autoimmunity is more common in females than in males. One possible explanation for this female preponderance may be the effect of oestrogens on the immune system. It has also been suggested that foetal microchimerism involving transfer of foetal cells into maternal tissue during pregnancy may play an important role.

Objective: We investigated the association between the presence of circulating thyroid autoantibodies and previous pregnancy, parity and the use of oral contraceptives (OCs) and hormone replacement therapy (HRT) in a population cohort.

Methods: We examined 3712 women randomly selected from the general population. Serum was analysed for thyroid peroxidase antibody (TPO-Ab) and thyroglobulin antibody (Tg-Ab) using assays based on an RIA technique (DYNO test). Data were analysed in logistic regression models to adjust for possible confounders. Women previously treated for thyroid disease or with pregnancy within 1 year prior to the study were excluded from the analyses.

Results: In both univariate and multivariate models and whether the presence of TPO-Ab and Tg-Ab was investigated alone or in combination, findings were negative with respect to an association between circulating thyroid antibodies and previous pregnancy, number of pregnancies, parity and previous abortion. There was no association between thyroid autoantibodies and use of OCs. Women aged 60-65 years receiving HRT now or previously had a lower prevalence of Tg-Ab (univariate, $P=0.01$; multivariate, $P=0.02$ ). No such association was observed between HRT and TPO-Ab.

Conclusion: In this population study there was no association between previous pregnancy, parity and thyroid antibodies, which argues against the role of microchimerism as a trigger of thyroid autoimmunity. Exogenous oestrogens may reduce aspects of autoimmunity.
\end{abstract}

European Journal of Endocrinology 154 39-45

\section{Introduction}

Autoimmune diseases are a poorly understood group of disorders. The regulatory malfunction of the immune system is often supposed to be secondary to a genetic predisposition currently thought to be multigenic (1). However, even in a genetically predisposed person, a triggering event is usually required for frank autoreactivity $(2,3)$. The character of this trigger is often unknown.

Autoimmune thyroiditis (AITD) is one of the most common autoimmune disorders. Like other thyroid diseases, AITD is more common in females than in males $(4,5)$. One possible explanation for this female predominance could be the effect of oestrogens on the immune system (6). Another possibility is that foetal microchimerism could contribute to the pathogenesis of AITD (6). This hypothesis is supported by the predominance of females but also by the fact that chronic graft-vs-host disease shares similarities with some autoimmune diseases (7).

Microchimerism is the presence of a mixture of a small number of cells from different individuals coexisting within tissue, including the peripheral blood. In foetal microchimerism, there is a transfer of cells or DNA from the foetus to the mother during pregnancy (8). Foetal-derived DNA has been found in maternal blood as early as in the first trimester (9) and up to 38 years after pregnancy (10). The foetal cells which remain in the maternal tissue after delivery may modu- 
late AITD by playing a role in antigen presentation. If foetal microchimerism contributes to thyroid autoimmunity in women there would be an association between previous pregnancy and the presence of thyroid autoimmunity. We investigated the association between the presence of thyroid autoantibodies (thyroid peroxidase antibody (TPO-Ab) and thyroglobulin antidody $(\mathrm{Tg}-\mathrm{Ab}))$ in the circulation and previous pregnancies, parities, abortions and use of oral contraceptives (OCs) and hormone replacement therapy (HRT) in a population cohort.

\section{Subjects and methods}

\section{Study population}

This study was part of the Danish Investigation of Iodine Intake and Thyroid Diseases (DanThyr), which is the official clinical monitor of the Danish iodine supplementation programme. The present investigation took place before the initiation of iodine supplementation. This study population represents the female part of the DanThyr cohort, which has been described in detail previously (11).

A sample of females within the following age groups, young adults (18-22 years), mid-gestational age (25-30 years), premenopausal $(40-45$ years) and postmenopausal (60-65 years) living in either Aalborg, Northern Jutland or Copenhagen, was drawn from the Civil Registration System. The subjects were given random numbers within each group with the help of computer software and were invited to the study examination in the order of the random numbers. The number of subjects invited in each group was adjusted throughout the study period to obtain uniform numbers of participants in all groups. The participants were invited by letter. When no response was received a new invitation was sent by mail. Of the 7286 subjects invited, $3712(50.9 \%)$ participated in the examination. The age distribution of the participants was homogenous in the two subcohorts.

Within the two geographically delimited areas the median iodine concentrations in spot urine were $45 \mu \mathrm{g} / \mathrm{l}$ (Aalborg) and $61 \mu \mathrm{g} / \mathrm{l}$ (Copenhagen) after the exclusion of subjects taking iodine supplementation (53 $\mu \mathrm{g} / \mathrm{l}$ and $68 \mu \mathrm{g} / \mathrm{l}$ in all subjects) (12).

\section{Data collection}

The participants answered questionnaires concerning previous treatment for thyroid disease and smoking habits and an obstetric anamnesis including the number of previous pregnancies, parities and abortions either spontaneous or provoked. Previous or present use of OCs and HRT was registered. Blood samples were collected.

\section{Laboratory procedures}

Serum TPO-Ab and Tg-Ab were both measured by RIA (DYNO test anti-TPO and DYNO test anti-Tg; BRAHMS Diagnostica, Berlin, Germany). The assays and evaluation of cut-off values have been described in detail previously (4). The following detection limits were used: $\mathrm{TPO}-\mathrm{Ab}>30 \mathrm{U} / \mathrm{l} ; \mathrm{Tg}-\mathrm{Ab}>20 \mathrm{U} / \mathrm{l}$, both corresponding to the functional sensitivity given by the manufacturer.

\section{Statistical analysis and distribution of variables}

All data processing was performed with SPSS 10.0 software (SPSS Inc. Chicago, Illinois, USA). The association between the presence of thyroid autoantibodies (TPO$\mathrm{Ab}$ and $\mathrm{Tg}-\mathrm{Ab}$ ) in serum and previous pregnancies, parities, abortions and use of OCs or HRT were analysed in logistic regression models to allow adjustment for possible confounding by other factors.

The prevalence of thyroid antibodies increases with age in women as does the number of previous pregnancies. Thus age would confound the evaluation of association between thyroid antibodies and previous pregnancy, and age group was included in all simple regression models. Because of the different distribution of the use of OCs and HRT and of smoking between the two regions and age groups, we included age group, smoking and region in the multivariate models.

Women who had been treated for thyroid disease $(n=207)$ or who were or had been pregnant within the last 12 months $(n=144)$ and cases with missing measurements of TPO-Ab or Tg-Ab $(n=78)$ were excluded, leaving 3283 cases for analysis. The analyses of the use of OCs included only women aged 18-45 years and the analyses of the use of HRT only women aged 60-65 years.

The distribution of some of the central variables is shown in Table 1. The level of significance was set at $5 \%$.

The study was approved by the regional Ethics Committees in Northern Jutland and Copenhagen and all participants gave written informed consent.

\section{Results}

The prevalence rates of $\mathrm{TPO}-\mathrm{Ab}$ and $\mathrm{Tg}-\mathrm{Ab}$ increased with increasing age (Table 2).

There was no association between the presence of thyroid autoantibodies (TPO-Ab and/or $\mathrm{Tg}-\mathrm{Ab}$ ) in serum and previous pregnancy, parity and provoked or spontaneous abortion (Fig. 1). In a multivariate model which included smoking and region we still found no association between TPO-Ab and/or Tg$\mathrm{Ab}$ and previous pregnancy, parity and abortion (previous pregnancy: OR (CI) 0.92 (0.72-1.17); parity: 1.02 (0.85-1.12); spontaneous abortion: 1.07 (0.83-1.38); provoked abortion: 0.99 (0.78-1.25)). 
Table 1 Distribution of variables in the 3283 women from the population studied.

\begin{tabular}{|c|c|c|c|c|c|}
\hline Variable & Parameter & $\begin{array}{c}\text { 18-22 years } \\
(n=897)\end{array}$ & $\begin{array}{c}25-30 \text { years } \\
(n=800)\end{array}$ & $\begin{array}{c}\text { 40-45 years } \\
(n=859)\end{array}$ & $\begin{array}{c}60-65 \text { years } \\
(n=727)\end{array}$ \\
\hline \multirow[t]{4}{*}{ Pregnancies } & None & 802 & 456 & 94 & 67 \\
\hline & One & 73 & 178 & 138 & 85 \\
\hline & Two & 16 & 103 & 264 & 210 \\
\hline & Three or more & 6 & 63 & 363 & 365 \\
\hline \multirow[t]{4}{*}{ Childbirths } & None & 878 & 590 & 148 & 90 \\
\hline & One & 14 & 125 & 211 & 109 \\
\hline & Two & 4 & 75 & 374 & 266 \\
\hline & Three or more & 1 & 10 & 126 & 262 \\
\hline \multirow[t]{2}{*}{ Spontaneous abortion } & Yes & 15 & 59 & 221 & 143 \\
\hline & No & 882 & 741 & 638 & 583 \\
\hline \multirow[t]{2}{*}{ Provoked abortion } & Yes & 73 & 185 & 312 & 166 \\
\hline & No & 824 & 615 & 547 & 561 \\
\hline \multirow[t]{2}{*}{ OC now } & Yes & 551 & 328 & 75 & - \\
\hline & No & 346 & 472 & 784 & - \\
\hline \multirow[t]{2}{*}{ OC ever } & Yes & 729 & 737 & 762 & - \\
\hline & No & 168 & 62 & 96 & - \\
\hline \multirow[t]{2}{*}{ HRT at menopause } & Yes & - & - & - & 277 \\
\hline & No & - & - & - & 423 \\
\hline \multirow[t]{2}{*}{ HRT now } & Yes & - & - & - & 140 \\
\hline & No & - & - & - & 556 \\
\hline
\end{tabular}

Missing values: women aged 25-30 years, OC ever: $n=1 ; 40-45$ years, OC ever: $n=1 ; 60-65$ years, spontaneous abortion: $n=1$; HRT at menopause: $n=27$; HRT now: $n=31$.

The risk of having thyroid antibodies (TPO-Ab and/or $\mathrm{Tg}-\mathrm{Ab}$ ) was similar in women who had never been pregnant compared with women with one or more previous pregnancies and in women who had never given birth compared with women with one or more previous childbirths (Fig. 2). However, in the simple model, women with one parity had a tendency for a lower risk of having thyroid autoantibodies measured in serum compared with nullipara (one/no parity, OR (CI): $0.81(0.60-1.10)$ and a significantly lower risk compared with women with two, three or more previous parities (one/two parities (OR (CI): 0.74 (0.55-0.98); one/ $\geq$ three parities: 0.67 (0.48-0.93). This tendency was confirmed in the multivariate model (one/two parities: OR (CI): 0.75 (0.56-1.0); one/ $\geq$ three parities: 0.69 (0.50-0.96)).

There was no association between previous pregnancy and elevated thyrotrophin (TSH) in women with TPO-Ab and/or Tg-Ab measured in the serum. However, in women without thyroid autoantibodies, elevated TSH was more frequent in women without any pregnancies compared with women with previous pregnancies when data were analysed in a logistic regression model adjusting for age group $(P=0.02)$.
The association between the use of OCs and thyroid autoantibodies (TPO-Ab and/or $\mathrm{Tg}$ - $\mathrm{Ab}$ ) was tested only in women aged $18-45$ years. We found no association between the present use of OCs and thyroid autoantibodies and ever having used OCs and thyroid autoantibodies either in simple logistic regression models (Fig. 3) or in multivariate models (present use of OC, OR (CI): 0.92 (0.72-1.17); ever having used OCs: $0.88(0.65-1.20))$.

The association between HRT and thyroid autoantibodies was tested in postmenopausal women aged 60-65 years. In a simple logistic regression model, we found a negative association between TPO-Ab and/or Tg-Ab in serum and use of HRT at menopause and between TPO-Ab and/or $\mathrm{Tg}-\mathrm{Ab}$ and present use of HRT (Fig. 4). When smoking and region were included in the multivariate models, the association between present use of HRT and thyroid autoantibodies was still significant (OR (CI): 0.63 (0.40-0.97). However, the association between use of HRT at menopause and the presence of TPO-Ab and/or Tg-Ab became statistically insignificant (OR (CI): $0.72(0.51-1.02)$ ).

To study the effect of HRT in more detail we tested the use of HRT at menopause against different

Table 2 Prevalence rates (\%) of TPO-Ab and Tg-Ab in women of various ages. Prevalence rate of different combinations of thyroid antibodies in the four groups of women with no current or previously treated thyroid disease or pregnancy within the last 12 months.

\begin{tabular}{lcccrr}
\hline Age group (years) & Number & TPO-Ab and/or Tg-Ab & TPO-Ab (\%) & Tg-Ab (\%) & TPO-Ab and Tg-Ab \\
\hline $18-22$ & 897 & 12.3 & 7.4 & 9.1 & 4.2 \\
$25-30$ & 800 & 18.2 & 12.0 & 14.0 & 7.8 \\
$40-45$ & 859 & 24.9 & 19.1 & 15.8 & 10.0 \\
$60-65$ & 727 & 29.7 & 21.7 & 20.0 & 12.0 \\
Total & 20.9 & 14.7 & 14.5 & 8.3 \\
\hline
\end{tabular}




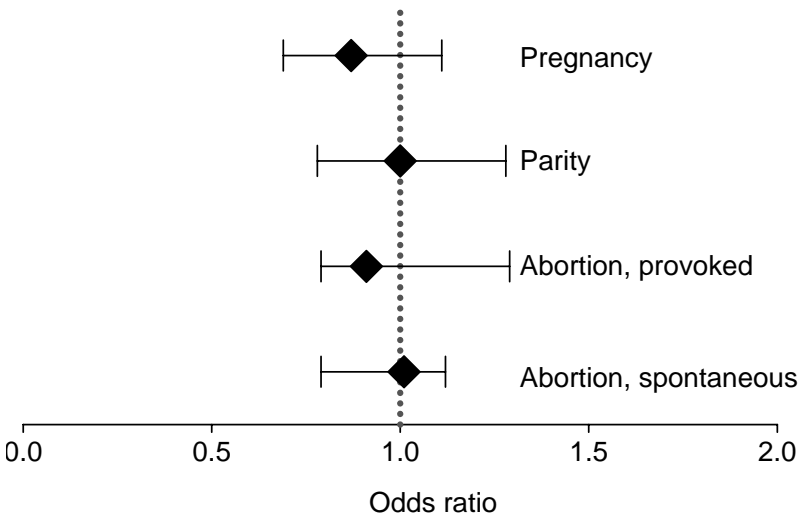

Figure 1 The association between thyroid autoantibodies (TPO$\mathrm{Ab}$ and/or Tg-Ab) in serum and previous pregnancy, parity and abortion; odds ratios (ORs) with 95\% confidence intervals (Cls) from four simple logistic regression models. No previous pregnancy, no parity and no abortion were the references. No statistically significant associations were present as 1 was included in all Cls. When data were tested in multivariate models the results were similar $(n=3283)$.

combinations of thyroid autoantibodies in serum (Fig. 5). There was no association between HRT and the presence of TPO-Ab, but a clear association between HRT and a low risk of having Tg-Ab (Fig. 5).

Sensitive antibody assays were used for this study and we included participants with low levels of thyroid antibodies in serum as the positive. To evaluate participants with higher levels of antibody only, all data were re-analysed in logistic regression models using $100 \mathrm{U} / \mathrm{ml}$ as cut-off for both the TPO-Ab and the Tg$\mathrm{Ab}$ assay. The prevalence rate of thyroid antibody above this level was: TPO-Ab and/or Tg-Ab; women aged 18-22 years: $6.7 \%$; 25-30 years: $10.5 \%$; 40-45 years: $14.9 \%$; $60-65$ years: $19.0 \%$.

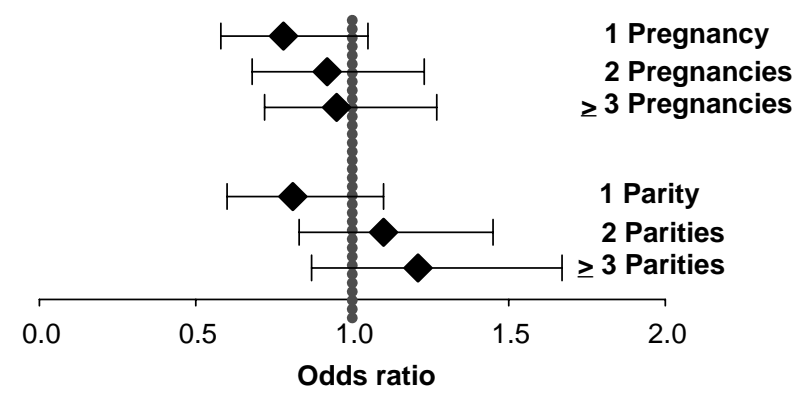

Figure 2 The association between TPO-Ab and/or Tg-Ab in serum and numbers of previous pregnancies and parities; ORs with $95 \% \mathrm{Cls}$ from two simple logistic regression models. No pregnancy and no parity were the references. The risk of having thyroid autoantibodies was the same in women who had never been pregnant compared with women with one or more previous pregnancies and in nullipara compared with women with one or more previous parities. Women with one parity had a significantly lower risk of having thyroid antibodies than women with two or more parities $(n=3283)$.

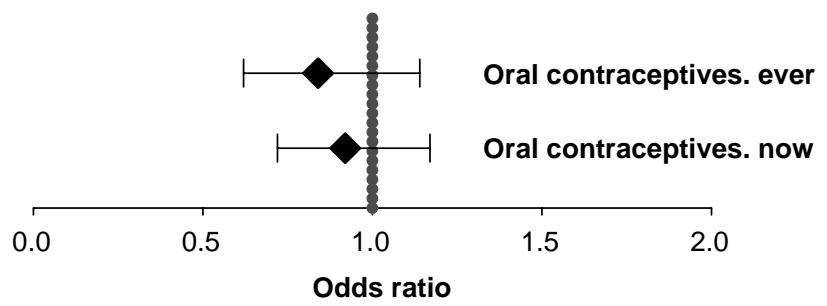

Figure 3 The association between having TPO-Ab and/or Tg-Ab measured in the serum and treatment with OCs ever or now. Figures are ORs with $95 \%$ Cls from two simple logistic regression models. No treatment with $\mathrm{OC}$ ever or now were the references. Only women aged $18-45$ years were included in the models $(n=2556)$. There was no association between thyroid autoantibodies and treatment with OCs ever or now as 1 was included in the confidence intervals. Similar results were seen when data were analysed in multivariate models.

Again we found no association between the presence of TPO-Ab and/or Tg-Ab in serum and previous pregnancy (OR (CI)): $0.94(0.70-1.26)$; previous parity: 1.02 (0.76-1.37); spontaneous abortion: 0.97 (0.71-1.31); provoked abortion: 1.06 (0.81-1.40); present treatment with OCs: $0.85(0.62-1.16)$; ever having used OCs: $0.83(0.57-1.20)$. The association between the presence of $\mathrm{Tg}-\mathrm{Ab}$ and the use of HRT at menopause became statistically insignificant.

\section{Discussion}

In this random sample of 3283 women from the general population without previous thyroid disease or pregnancies 1 year prior to the study, we found no association between the presence of circulating thyroid autoantibodies and previous pregnancy, parity and abortion. There was no association between thyroid autoantibodies and the use of OCs currently or ever having been used. Women treated with HRT had a lower risk of having thyroid autoantibodies compared

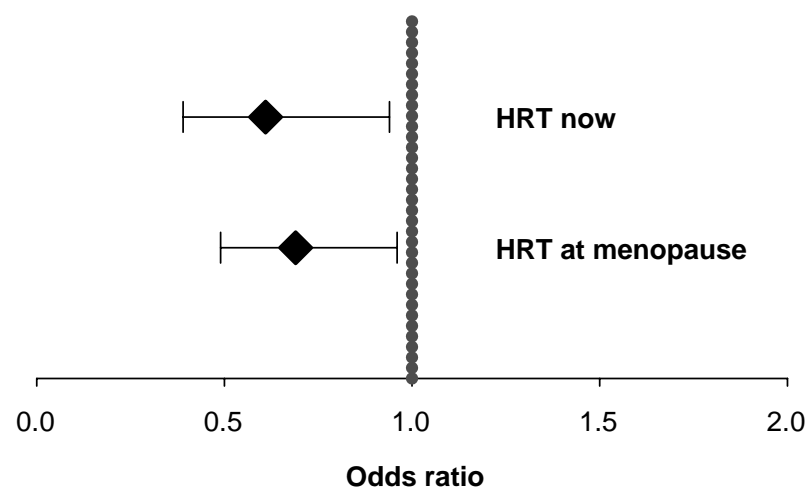

Figure 4 The association between TPO-Ab and/or Tg-Ab in the serum and use of HRT now or at menopause in 727 postmenopausal women aged 60-65 years; ORs with $95 \%$ Cls from two simple logistic regression models. No HRT at menopause or now were the references. 


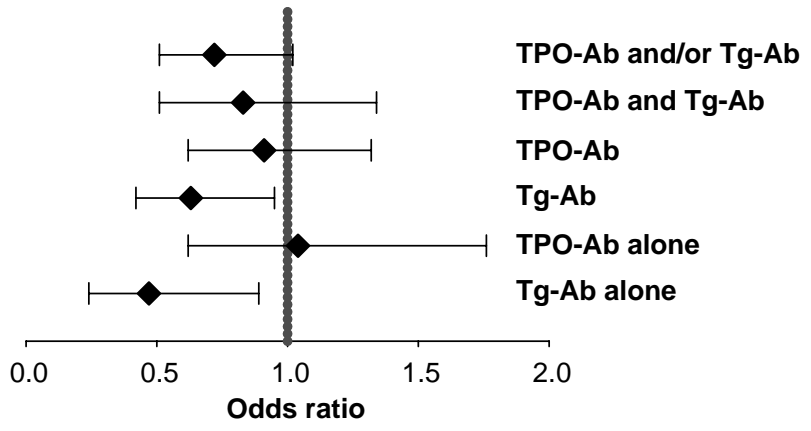

Figure 5 The association between different combinations of thyroid autoantibodies in serum and use of HRT at menopause. Figures are ORs with $95 \%$ Cls from six multivariate logistic regression models. Participants without the specific antibody combination are used as a reference in each case. HRT was negatively associated with $\mathrm{Tg}-\mathrm{Ab}$ measured either alone or together with TPO-Ab. There was no association between use of HRT at menopause and the presence of TPO-Ab.

with women who were not treated with HRT. This association was confined to $\mathrm{Tg}-\mathrm{Ab}$ whereas there was no association with TPO-Ab.

Circulating thyroid autoantibodies are more common in females than in males $(4,5)$. The reason for this female preponderance is unclear. Hormonal and genetic factors may play a role as girls with Turners's syndrome (XO karyotype) are more often affected by thyroid autoimmunity (13). The high prevalence of thyroid autoimmunity in women after child-bearing age suggests that pregnancy-related factors could have an influence on the development of AITD. These factors could be hormonal, but it has also been suggested that foetal microchimerism might be important in the pathogenesis of AITD and other autoimmune diseases including scleroderma $(6,14,15)$.

A relatively simple method to detect microchimerism is to demonstrate male-specific gene markers in women who have been pregnant with a male child (16). Malespecific gene markers have been demonstrated in peripheral blood from 30-34\% of healthy women with a known past male pregnancy, indicating that microchimerism often occurs in normal pregnancies $(17,18)$. The circumstances necessary for these foetal-derived cells to become established and expand and furthermore migrate to interact with multiple tissues including the thyroid are unknown. It is likely, however, that genetic, environmental and other yet-unidentified factors combine to determine the persistence of the foetal tissue (7).

If microchimerism is important in the pathogenesis of AITD the prevalence of thyroid autoantibodies (TPO-Ab and/or Tg-Ab) would be expected to be higher in women with previous pregnancies compared with women without any pregnancies. The present study does not support this idea.

Relatively few studies have tested the association between microchimerism and AITD, and the results are not equivocal. In a case-control study of 89 women with Hashimoto's thyroiditis, Phillips et al. (19) found no evidence of an association between Hashimoto's thyroiditis and previous parity, and no trend to an increasing disease risk with an increasing number of parities. On the other hand, some studies support the hypothesis of a link between microchimerism and AITD. Klintschar et al. (20) detected microchimerism in the thyroid in eight out of 17 patients suffering from Hashimoto's disease compared with only one out of 25 controls with nodular goitre. The study group and control group had comparable numbers of pregnancies and sons. In a study by Renne et al. (21) of 49 women, who all had sons, microchimerism was found in the thyroid from 23 out of 40 women with AITD (Hashimoto's disease: $n=25$; Graves'disease: $n=15$ ), compared with two out of nine women with thyroid adenoma. Srivatsa et al. (22) detected microchimerism in 12 out of 20 thyroids from women who had sons and who had undergone thyroidectomy for various thyroid diseases (nodular goitre: $n=9$; AITD: $n=11$ ). Four out of the 12 thyroids containing male cells were classified as nodular goitre. In only eight out of the 11 thyroids classified as AITD could microchimerism be detected. In the control group, consisting of eight thyroids taken from women who had died from other disorders, microchimerism was detected in none. Accordingly, at least three studies suggest a relation between microchimerism and AITD $(20-22)$ or at least between microchimerism and thyroid disease (22). However, the role of microchimerism is unlikely to be essential for developing AITD as microchimerism was not detected in $25-48 \%$ of the patients with AITD. It is possible that the difference in the prevalence of microchimerism between patients and the control groups might result from general immunological differences and perhaps differences in the leakiness of the placenta during pregnancy. Therefore this microchimerism might be not the cause for - but the consequence of - a pre-existing subclinical autoimmune disease involving the placenta (20). It might also be speculated that a thyroid affected by autoimmunity would give better possibilities of survival of foetal cells trapped in the thyroid during pregnancy.

A number of studies have dealt with microchimerism in women with scleroderma. Most of the studies have not been able to show an increased prevalence of microchimerism in women suffering from scleroderma compared with controls. In a hospital based study of 46 women with scleroderma, Pisa et al. (23) even found a reduced risk of scleroderma with previous parity. The risk decreased with increasing numbers of children. Nelson et al. (15) demonstrated that the prevalence of male cells was not higher in women with scleroderma compared with controls. On the other hand, the concentration of male DNA in women with scleroderma was higher than in healthy controls. 
Immune suppression during pregnancy suggests that high levels of endogenous oestradiol may prevent autoimmunity. There are relatively few studies concerning the association between exogenous oestrogens and thyroid autoimmunity and the results are dissimilar. In the present study, we found no association between OCs and thyroid autoantibodies, whereas HRT was negatively associated with $\mathrm{Tg}-\mathrm{Ab}$ but not with TPO-Ab. There are, however, some studies suggesting that OCs could be protective against the development of AITD. In a large study of 46000 women, the overall prevalence of hypo- and hyperthyroidism was less frequent among OC users than controls (24). Vestergaard et al. (25) found that ever having used OCs was associated with a slightly lower risk of Graves'disease but not of Hashimoto's disease. In a cohort study of 803 subjects at risk of developing AITD, Strieder et al. (26) found that the use of oestrogen was negatively correlated with the presence of TPO-Ab. In a follow-up study of healthy middle-aged women, Massoudi et al. (27) found no difference in antibody level in postmenopausal women using HRT compared with women who did not (27).

These discrepancies in association between thyroid autoantibodies and exogenous oestrogens may be explained by the different methods applied and especially differences in the sensitivity of the antibody assays used.

In conclusion, it has been shown in a number of studies that foetal cells, circulating in the pregnant woman, may be trapped and survive for years in the thyroid of the mother. However, we find no evidence that such a mechanism is of importance for the high prevalence of thyroid autoimmunity in women in the population.

\section{Acknowledgements}

This study was a part of The Danish Investigation of Iodine Intake and Thyroid Diseases (DanThyr), which is supported by grants from the Danish Medical Foundation, the 1991 Pharmacy Foundation, North Jutland County Research Foundation, Tømmerhandler Wilhelm Bangs Foundation and Copenhagen Hospital Corporation Research Foundation.

\section{References}

1 Davidson A \& Diamond B. Autoimmune diseases. New England Journal of Medicine (Review) 2001345 340-350.

2 Safran M, Paul TL, Roti E \& Braverman LE. Environmental factors affecting autoimmune thyroid disease. Endocrinology and Metabolism Clinics of North America $198716302-327$.

3 Tomer Y \& Davies TF. Infection, thyroid disease and autoimmunity. Endocrine Reviews 199314 107-120.

4 Bülow Pedersen I, Knudsen N, Jørgensen T, Perrild H, Ovesen L \& Laurberg P. Thyroid peroxidase and thyroglobulin autoantibodies in a large survey of populations with mild and moderate iodine deficiency. Clinical Endocrinology 200358 36-42.
5 Tunbridge WM, Evered DC, Hall R, Appleton D, Brewis M, Clark F, Grimley Evans J, Young E, Bird T \& Smith RA. The spectrum of thyroid disease in a community: the Wickham survey. Clinical Endocrinology 19777 481-493.

6 Prummel MF, Strieder T \& Wiersinga WM. The environment and autoimmune thyroid disease. European Journal of Endocrinology $2004150605-618$.

7 Sarkar K \& Miller FW. Possible roles and determinants of microchimerism in autoimmune and other disorders. Autoimmunity Reviews 20043 454-463.

8 Scroder J. Transplacental passage of blood cells. Journal of Medical Genetics 197512 230-242.

9 O’Donoghue K, Choolani M, Chan J, de la Fuente J, Kumar S, Campagnoli C, Bennett PR, Roberts IAG \& Fisk NM. Identification of fetal mesenchymal stem cells in maternal blood: implications for non-invasive prenatal diagnosis. Molecular Human Reproduction 20039 497-502.

10 Evans PC, Lambert N, Maloney S, Furst DE, Moore JM \& Nelson JL. Long-term fetal microchimerism in peripheral blood mononuclear cell subsets in healthy women and women with scleroderma. Blood 199993 2033-2037.

11 Knudsen N, Bülow I, Jørgensen T, Laurberg P, Ovesen L \& Perrild H. Goitre prevalence and thyroid abnormalities at ultrasonography: a comparative epidemiological study in two regions with slightly different iodine status. Clinical Endocrinology 2000 53 479-485.

12 Rasmussen LB, Ovesen L, Bülow I, Jørgensen T, Knudsen N, Laurberg P \& Perrild H. Dietary iodine intake and urinary iodine excretion in a Danish population: effect of geography, supplements and food choice. British Journal of Nutrition 200287 61-69.

13 Elsheikh M, Wass JAH \& Conway GS. Autoimmune thyroid syndrome in women with Turner's syndrome - the association with karytype. Clinical Endocrinology 200155 223-226.

14 Badenhoop K. Intrathyroidal microchimerism in Graves' disease or Hashimoto's thyroiditis: regulation of tolerance or alloimmunity by fetal-maternal immune interactions? European Journal of Endocrinology 2004150 421-423.

15 Nelson JL, Furst DE, Maloney S, Gooley T, Evans PC, Smith A, Bean MA, Ober C \& Bianchi DW. Microchimerism and HLA-compatible relationships of pregnancy in scleroderma. Lancet 1998 $351559-562$.

16 Ando T \& Davies TF. Self-recognition and the role of fetal microchimerism. Best Practice and Research Clinical Endocrinology and Metabolism 200418 197-211.

17 Ando T, Imaizumi M, Graves PN, Unger P \& Davies TF. Intrathyroidal fetal microchimerism in Graves' disease. Journal of Clinical Endocrinology and Metabolism 200287 3315-3320.

18 Adams KM, Lambert NC, Heimfeld S, Tylee TS, Pang JM, Erickson TD \& Nelson JL. Male DNA in female donor apheresis and CD34-enriched products. Blood 2003102 3845-3847.

19 Phillips DI, Lazarus JH \& Butland BK. The influence of pregnancy and reproductive span on the occurrence of autoimmune thyroiditis. Clinical Endocrinology 199032 301-306.

20 Klintschar M, Schwaiger P, Mannweiler S, Regauer S \& Kleiber M. Evidence of fetal microchimerism in Hashimoto's thyroiditis. Journal of Clinical Endocrinology and Metabolism 200186 2494-2498.

21 Renne C, Ramos Lopez E, Steimle-Grauer SA, Ziolkowski P, Pani MA, Luther C, Holzer K, Encke A, Wahl RA, Bechstein WO, Usadel KH, Hansmann ML \& Badenhoop K. Thyroid fetal male microchimerism in mothers with thyroid disorders: presence of Y-chromosomal immunofluorescence in thyroid-infiltrating lymphocytes is more prevalent in Hashimoto's thyroiditis and Graves' disease than in follicular adenomas. Journal of Clinical Endocrinology and Metabolism 200489 5810-5814.

22 Srivatsa B, Srivatsa S, Johnson KL, Samura O, Lee SL \& Bianchi DW. Microchimerism of presumed fetal orign in thyroid specimens from women: a case-control study. Lancet $2001 \mathbf{3 5 8}$ 2034-2038.

23 Pisa FE, Bovenzi M, Romeo L, Tonello A, Biasi D, Bambara LM, Betta A \& Barbone F. Reproductive factors and risk of scleroderma. 
An Italian case control study. Arthritis and Rheumatism 200246 $451-456$.

24 Frank P \& Kay CR. Incidence of thyroid disease associated with oral contraceptives. British Medical Journal 197821531.

25 Vestergaard P, Rejnmark L, Weeke J, Hoeck HC, Nielsen HK, Rungby J, Laurberg P \& Mosekilde L. Smoking as a risk factor for Graves' disease, toxic nodular goiter, and autoimmune hypothyroidism. Thyroid 200212 69-75.

26 Strieder TGA, Prummel MF, Tijssen JGP, Endert E \& Wiersinga WM. Risk factors for and prevalence of thyroid disorders in a cross-sectional study among healthy female relatives of patients with autoimmune thyroid disease. Findings from the
Thyroid Study of Healthy Women. Clinical Endocrinology 2003 59 396-401.

27 Massoudi MS, Meilahn EN, Orchard TJ, Foley TP, Kuller LH, Costantino JP \& Buhari AM. Prevalence of thyroid antibodies among healthy middle-aged women. Annals of Epidemiology $19955229-233$.

Received 31 July 2005

Accepted 14 October 2005 